\title{
Characterization of nanoscaled films on flat and grating substrates as some elements of plasmonics
}

\author{
N.L. Dmitruk, O.I. Mayeva, A.V. Korovin, S.V. Mamykin, M.V. Sosnova, O.B. Yastrubchak \\ V. Lashkaryov Institute of Semiconductor Physics, NAS of Ukraine, 41, prospect Nauky, 03028 Kyiv, Ukraine \\ Phone: (38044) 52565 46; fax: (83044) 52582 43; e-mail: Sosnova@isp.kiev.ua
}

\begin{abstract}
The optical properties of multilayer structures consisting of dielectric, conductivity-oxide and nanoscaled metal layers, deposited on the planar substrates (witness samples) and surface relief ones (diffraction gratings) with micro- and nanoscale sizes, are investigated by AFM, spectral ellipsometry (SE), and photometric techniques. The SE-measured parameters are related to actual characteristics of the layers when specified the model of their near-surface regions. Using a parametrization of the layer dielectric function versus the wavelength and a fitting procedure, the dielectric parameters are determined. It is shown that the optical constants are affected by both the substrate morphology and the adjacent medium. Preliminary data about the influence of isolated particle plasmon excitations in 2D-substrates with the top nanoscaled Au layer on its optical properties are presented.
\end{abstract}

Keywords: diffraction grating, nanoscaled film, surface plasmon polariton, plasmonics.

Manuscript received 04.04.07; accepted for publication 24.04.07; published online 19.10.07.

\section{Introduction}

The effect of the resonant excitation of surface plasmon polaritons (SPPs) or guided waves in optical multilayer structures attracts a special attention for both basic researches and possible practical applications $[1,2]$. SPP waves coupled with collective electron oscillations can be used as information carriers in highly integrated photonic devices with structural elements smaller than a light wavelength (e.g., nanostructured metal films). Such a technique is effective to enable active SPP signals to be manipulated and guided. In such a context, we speak about "plasmonics".

Numerous SPPs applications can be found in the area of chemo- and biosensors, as even some modification of a metal surface results in a change of the SPP resonant conditions and allows monitoring the environmental conditions, to which the surface is exposed. Furthermore, the strong enhancement of the SPP local field at the surface and an extremely high sensitivity of the SPP dispersion law to optical properties of the interface make them a unique probe of the properties of surfaces and ultrathin films. For the greater sensitivity and more flexible design of SPP devices [35], the following steps could be useful: (i) inclusion of an additional covering and/or intermediate dielectric $\left(\mathrm{SiO}_{\mathrm{x}}\right)$ and/or conducting (ITO) tuning layers; (ii) changing the dielectric function of the metal at the interface by means of a nanoscale structural modification [6] of it; and (iii) using a diffraction grating (DG) as the input-coupling component to excite SPPs in the metallic system.

For the design of an optical multilayer system, it is important to know the optical constants of all the materials in use. It is a fact that, for most of today's standard deposition processes, the optical constants of the resulting films can vary with the process parameters [7]. It is well known from numerous fundamental thin film experiments that the substrate can have a significant influence on the optical properties (the refraction index, reflectivity, light scattering, and SPP excitation) of a coating deposited on it.

The main goal of this work is to characterize each technological step and optical properties of each layer used with the aim to construct an SPP device with the best parameters.

In this paper, we deal with the post deposition characterization of multilayer structures by $\mathrm{SE}$ and photometric technique. Also, SPP spectroscopy can be used as a tool to study optical properties of both laterally homogeneous and inhomogeneous metal (nanoscaled), dielectric (spacer, cover), and conducting films deposited on a planar or corrugated substrate which supports the SPP modes. 


\section{Sample preparation}

Surface relief DGs were prepared on GaAs (100) substrates by holographic etching described elsewhere [3]. Layered structures were produced by thermal evaporation of thin metal $(\mathrm{Au})$ and dielectric $\left(\mathrm{SiO}_{\mathrm{x}}\right)$ films as well as by magnetron sputtering of ITO on the processed semiconductor (GaAs) substrate at room temperature (RT). Then ITO films were annealed at $350^{\circ} \mathrm{C}$ for $30 \mathrm{~min}$ in vacuum to reduce their resistivity. Films of $\mathrm{SiO}_{x}$ were prepared by thermal evaporation of silicon monoxide. Substrates were placed both perpendicularly $\left(\mathrm{ITO}, \mathrm{SiO}_{\mathrm{x}}\right)$ and at an angle of $70{ }^{\circ} \mathrm{C}$ for the deposition of $\mathrm{Au}$ island films (measured from the surface normal). Both conductive (ITO) and dielectric $\left(\mathrm{SiO}_{\mathrm{x}}\right)$ oxide films were deposited also onto a flat microscope glass slide, the $\mathrm{GaAs}$ substrate (treated in the same solution as for holographic etching), and the processed semiconductor substrate (DG) in one deposition run.

One part of the DG substrate has been masked by a glass slide after deposition of an ITO layer. The small portion of the second part was etched to remove ITO and to leave the GaAs surface uncoated. Then gold was thermally evaporated in a high vacuum chamber at an oblique angle on the both parts of the DG sample. Deposition was performed at a rate of $10 \mathrm{~nm} / \mathrm{s}$. A quartz crystal was used to monitor both the evaporation rate and the thickness of the metal film. In this way, some variation in the thickness of a gold film ( 3 and $12 \mathrm{~nm}$ ) was achieved using the same fabrication conditions. Such a deposition process allows one to realize various measurements of multilayer heterostructures on the same DG. Four representative multilayer heterostructures (see Table 1) were prepared and assigned to numbers $S 1, S 2$, S3, S4, respectively, for the following measurements. With our procedure, the successive deposition of different layers with specific and tailored features on DGs along with witness sample $\mathrm{Au}(12 \mathrm{~nm}) /$ glass would permit the fabrication of thin films with variable optical properties.

\section{Characterization techniques}

In our earlier papers $[3,5]$, we have published some results on the determination of the optical constants of thin metal films, deposited on the flat and corrugated (DG) substrates, resulted from the optical monitoring of multilayer structures using SE. In the latter case, the

Table 1. Multilayer heterostructures under study.

\begin{tabular}{|c|c|c|c|c|c|}
\hline Structures & S1 & S2 & S3 & S4 \\
\hline \multicolumn{2}{|c|}{$\mathrm{GaAs}$} & $\mathrm{DG}^{*}$ & $\mathrm{DG}^{*}$ & $\mathrm{DG}^{*}$ & $\mathrm{DG}^{*}$ \\
\hline \multirow{2}{*}{} & $\mathrm{ITO}$ & $160 \mathrm{~nm}$ & - & $160 \mathrm{~nm}$ & $160 \mathrm{~nm}$ \\
\cline { 2 - 5 } & $\mathrm{Au}$ & - & $12 \mathrm{~nm}$ & $12 \mathrm{~nm}$ & $3 \mathrm{~nm}$ \\
\hline
\end{tabular}

${ }^{*}$ Grating period is $500 \mathrm{~nm}$. measurements of the ellipsometric angles $(\psi, \Delta)$ in the spectral region out of SPR were employed. The effect of a rough surface may be manifested as an increase in the effective thickness of intrinsic oxide [5]. Ellipsometric measurements were performed using a computercontrolled variable angle of an incident spectroscopic ellipsometer of the rotating analyzer type (VASE, J.A. Woollam Company). The basic measurements were made in the photon energy range $1.24 \ldots 5.0 \mathrm{eV}$ with the $0.02-\mathrm{eV}$ intervals at different incident angles. For the analysis, the VASE software WVASE $32^{\mathrm{TM}}$ was used.

The measurements of the morphological and statistical features of the samples surfaces under investigation were characterized using AFM (NanoScope ${ }^{\mathrm{TM}}$ III A controller and Dimension ${ }^{\mathrm{TM}} 5000$ stage system) in the contact-mode regime with a scan size of 1 and $5 \mu \mathrm{m}^{2}$. Figs. 1-4 including AFM-images demonstrate the features of the final structures depending upon the starting conditions of the growth of films defined by various types of the substrate. These conditions modify the condensation process from the gas phase, determine a microstructure of deposited films, and, as a consequence, their optical properties. Moreover, the film microstructure is the common factor in the optimization of almost all the film properties [8]. As a judged from the visual inspection of sectioning (Fig. 2a,b) of surfaces along the various directions (made by perpendicularly or in parallel to the grating's rules), grating's surface consists of micro- and nanogrooved regions. The former are a result of the holographic etching of the GaAs substrate and has DG (ruled) microstructure. For the latter, images of the same surface on the nanometer scale reveal a morphology of the quasi-grating type resulted from the anisotropic etching of the GaAs (100) substrate [9]. It is a system of approximately parallel grooves with the period value which increases with the etching duration. As was shown in our work [9], a real micro- (or nano-) relief of the quasi-grating type is composed of a random distribution of certain-type gratings, i.e. it is intermediate between the periodic and perfectly random ones. Existing some maxima revealed by the in-depth analysis (Fig. 3) are characteristic of a similar relief.

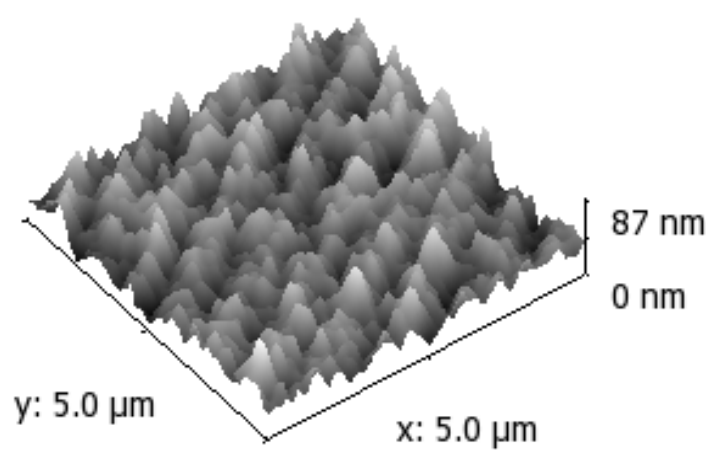

Fig. 1. AFM-image for scan areas $5 \times 5 \mu \mathrm{m}^{2}$ to sample $S 1$ (ITO/GaAs (DG). 

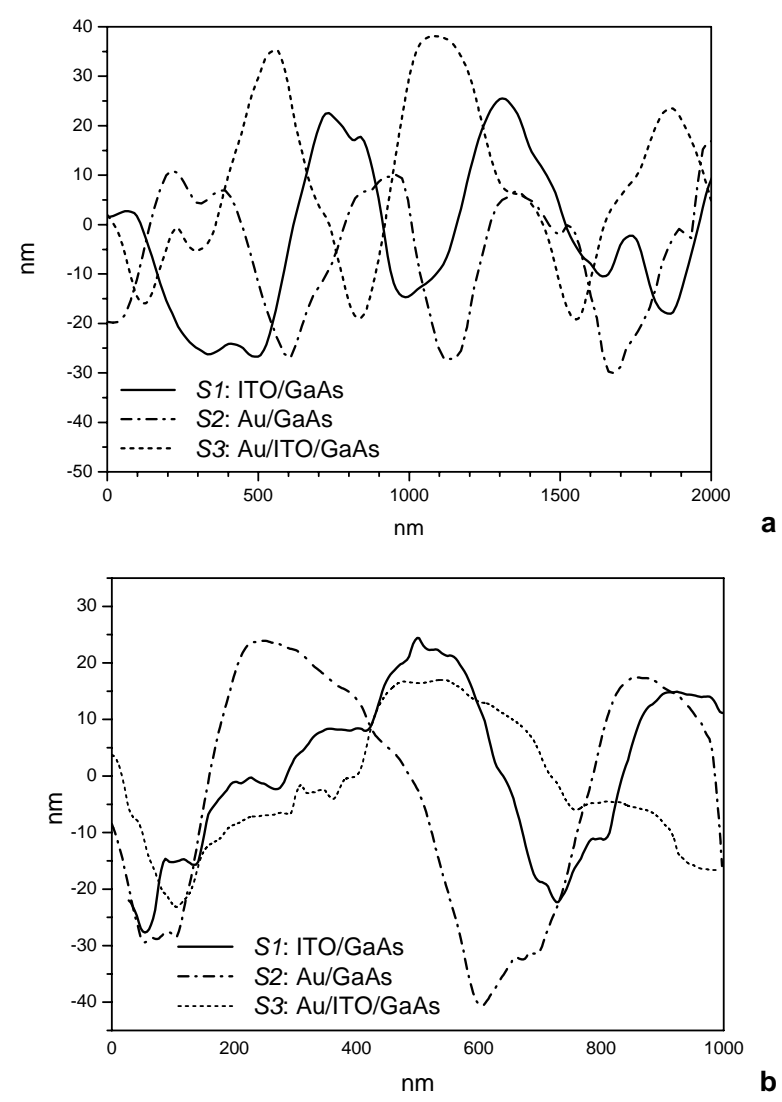

Fig. 2. Section analysis of the samples investigated for scan areas $5 \times 5 \mu^{2}$ (a) and $1 \times 1 \mu^{2}$ (b). The direction of the sectioning is perpendicular to grooves and demonstrates the profile changing due to different substrate morphologies.

We analyzed also the evolution of surface features which occurs under consecutive deposition of the abovementioned layers by using the method of power spectral density analysis (PSD). PSD is the Fourier transformation of the surface height (profile), from which the intensity spectrum of various profile harmonics is calculated (Fig. 4). Some periods are seen such as the fundamental one $(0.5 \mu \mathrm{m})$ and others.

When designing the multilayer structures, one introduces non-identity in the properties of deposited metal films: an $\mathrm{Au}$ thin film was evaporated onto the GaAs corrugated substrate covered (or not) by an ITO layer and oriented at an angle of $\sim 70^{\circ}$ (measured from the target surface normal) under the oblique-angle growth. Under the oblique-angle growth, the incident particles of an evaporated material are preferentially deposited on the top of substrate features with larger height due to the shadowing effect. This preferential growth gives rise to the formation of $\mathrm{Au}$ aggregates (island nanostructures). Depending upon the porosity and roughness of the underlying substrate, a modification of the initial growth conditions may occurs. As a result, the final microstructure of the deposited film will depend on a very delicate balance of different factors.
Table 2. Characteristics of the different structures under study (scan area $1 \times 1 \mu \mathrm{m}^{2}$ ).

\begin{tabular}{|l|c|c|c|}
\hline Structures & $\begin{array}{c}\boldsymbol{R}_{\boldsymbol{a}}(\mathrm{nm})- \\
\text { arithmetic } \\
\text { average of the } \\
\text { absolute va- } \\
\text { lues of height } \\
\text { deviations }\end{array}$ & $\begin{array}{c}\text { rms }(\mathrm{nm})- \\
\text { root mean } \\
\text { square } \\
\text { average } \\
\text { of height } \\
\text { deviations }\end{array}$ & $\begin{array}{c}\boldsymbol{h}(\mathrm{nm})- \\
\text { average } \\
\text { of heights }\end{array}$ \\
\hline \multirow{2}{*}{ S1: ITO/GaAs } & $10.91^{*}$ & $13.42^{*}$ & $40.68^{*}$ \\
\cline { 2 - 4 } & 10.81 & 13.53 & 42.21 \\
\hline S2: Au/GaAs & 16.31 & 19.61 & 51.28 \\
\hline $\begin{array}{l}\text { S3: } \\
\text { Au/ITO/GaAs }\end{array}$ & 11.42 & 9.18 & 35.41 \\
* scan area $5 \times 5 \mathrm{~m}^{2}$ & & \\
\hline
\end{tabular}

Structures S2 and S3 are obtained under identical conditions of $\mathrm{Au}$ evaporation, but display different microstructures (see Table 2). By comparing the morphological (Figs. 1,2) and statistical characteristics listed in Table 2, it is clearly seen that structure S2 is more corrugated than structure S3. The AFM-images of structures S2 and S3 are not shown for economy of

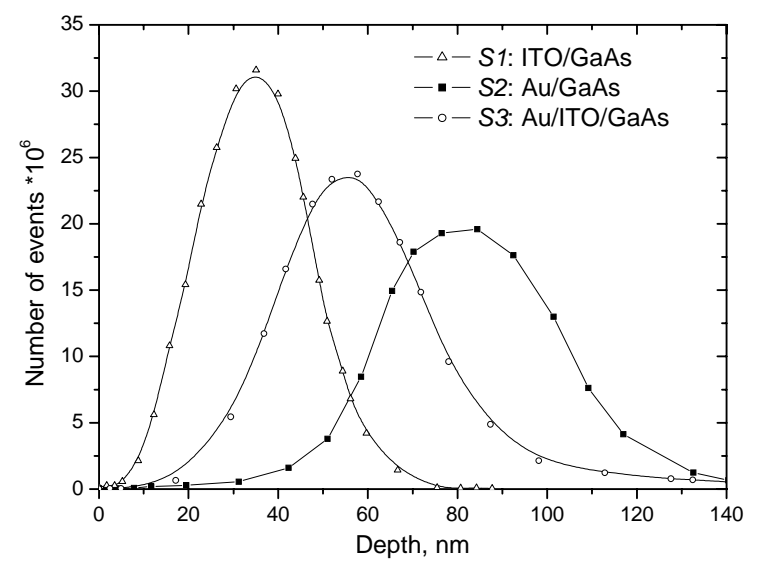

Fig. 3. Depth analysis for the samples; scan areas $5 \times 5 \mu \mathrm{m}^{2}$.

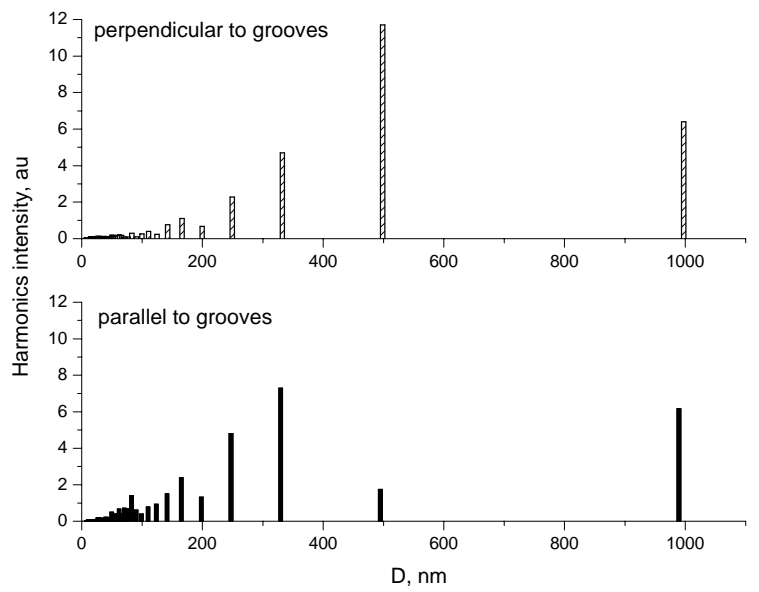

Fig. 4. Fourier harmonics spectra for DG profiles for the directions perpendicular and parallel to grooves. 
place. The results of the in-depth analysis depicted in Fig. 3 shows that the mean size of aggregates is larger for S2 than that for S3, although the size dispersion profiles are similar in both cases. We note that the size distribution is narrower for S3 than that for $S 2$.

\section{Parametrization of layer dielectric functions}

For the characterization of layers, the VASE technique based on measurements of ellipsometric angles $\psi$ and $\Delta$ was employed.

To relate the SE-measured parameters to actual characteristics of the constituents of multilayer structures designed, a model of near-surface regions (from which the Fresnel reflection coefficients are calculated) was specified. The proper multilayer stack calculation routine was performed. The optical functions of individual layers were determined by using both the parametrization of the layer dielectric function versus the wavelength and a fitting procedure to determine the individual parameters. For the material's optical properties which depend on deposition conditions, it can be made by various ways.

1. Approximation of semi-infinitive medium. As is known [10], the complex dielectric constant $\varepsilon$ of a film can be determined as

$$
\varepsilon=\varepsilon_{1}+i \varepsilon_{2}=\left(\frac{1-\rho}{1+\rho}\right)^{2} \sin ^{2} \theta \cdot \operatorname{tg}^{2} \theta,
$$

where $\rho=r_{p} / r_{s}=\operatorname{tg} \psi e^{i \Delta} ; \theta$ is the angle of incidence of the probing light; $r_{p}, r_{s}$ are, respectively, the complex Fresnel reflection coefficients for $p$ - and $s$-polarized light; $\varepsilon_{1}=n^{2}-k^{2}, \quad \varepsilon_{2}=2 n k$, and $n$ and $k$ are, respectively, the real and imaginary parts of the complex index of refraction $N: N=n-i k$.

2. Effective medium theories. The optical properties of thin films with complicated structure have been successfully modeled with the help of effective medium approximation (EMA) theories, typically the MaxwellGarnett (see, for example [11]) or Bruggeman [12] one. The latter is more frequently used (see, e.g., [3]), because it is applicable for any value of the filling factor.

3. The Sellmeier model. The Bruggeman-EMA considers the material as constituted by different macroscopic phases characterized by their bulk dielectric functions. In the case of a mixture on the atomic scale ( $\mathrm{Si}-\mathrm{Si}$ and $\mathrm{Si}-\mathrm{O}$ for $\mathrm{SiO}_{\mathrm{x}}$, for example), the Sellmeier model (see, e.g., [13]) is adopted, because it is quite good at reproducing the dispersion of the refraction index in the transparent region. According to this model, the dispersion formulas for $n$ and $k$ can be written as

$$
n^{2}(\lambda)=1+\frac{A}{1-\left(\frac{B}{\lambda}\right)^{2}} ; k(\lambda)=\frac{1}{n(\lambda)} \cdot \frac{C}{D \lambda+\frac{E}{\lambda}+\frac{1}{\lambda^{3}}},
$$

where $A, B, C, D$, and $E$ are fitting parameters, and $\lambda$ is the wavelength.
4. Lorentz oscillators. The dielectric functions of thin solid (especially compositionally graded) films are often simulated, by using the Lorentz oscillator model [14], as

$$
\widetilde{\varepsilon}=\varepsilon_{1}+i \varepsilon_{2}=\varepsilon_{\infty}+\sum_{j=1}^{N} \frac{f_{j} \omega_{0 j}^{2}}{\omega_{0 j}^{2}-\omega^{2}+i \Gamma_{j} \omega} .
$$

In this expression, $\omega$ is the photon frequency, $\varepsilon_{\infty}$ is the high-frequency dielectric constant, $\Gamma_{j}$ is the linewidth of the $j$-th oscillator, $f_{j}$ is the oscillator strength parameter, and $\omega_{0 j}$ are the resonance frequencies of oscillators.

5. The ellipsometric analysis performed by solving the Fresnel equations for $n, k$, and $d$ on the wavelengthby-wavelength basis (inhomogeneous layers). In the case of inhomogeneous overlayers (lossy or dielectric layers deposited onto the DG-substrate), it is essential that the optical signal contribution of the constituent of interest can be identified. As distinct from the discrete (or multilayer) description of the refractive index variation of an ITO layer deposited onto the flat GaAs substrate, the quasi-continuous-medium approximation was employed for the description of unintentional index gradients which arise from a microstructure that is developed in ITO films evaporated on the DG surfaces (S1). As a new thin film is evaporated (S2 and S3), the structure of the entire film stack became very complex. Various factors, such as surface roughness, lateral film non-uniformity, and other microscopic interfacial effects, make a physically reasonable modeling extremely difficult. Therefore, the Bruggeman-EMA yields the acceptable fits to measured data not always. It can be indicated that the inhomogeneity indeed exists. Without additional analytical technique which gives the correct information on the chemical nature of a substrate, to obtain the effective values of optical constants, we were only able to fit the data on the wavelength-by-wavelength basis.

6. Theoretical analysis of optical properties through the reflectance/transmittance (angular and spectral) characteristics of multilayer structures. To obtain the layer parameters of the multilayer structures, the least-square technique is used. This method comprises the minimization of a functional which is the sum of mean-squared differences between the calculated and measured angular and spectral transmittance/ reflectance data. Thus, $n$ and $k$ are the parameters of a functional, for which we will determine a global minimum. A peculiarity of the functional is the occurrence of both a global minimum and local minima. So, the well-known method of gradients (or its modification, the method of conjugate gradients, see, e.g., [15]) is incorrect in this case, because a local minimum can be found instead of the global one. However, in our case of parametrization (two or three parameters, if the layer thickness should be considered), a search for the global minimum can be performed by the simple selection of $n$ and $k$ within the beforehand 
taken region. For a grating, the effect of a complicated periodic relief should be considered with the help of the differential formalism method (e.g., the C-method developed in [16]). In the case of the s-polarization of light (when the electric vector of an incident wave is directed in parallel to the direction of grooves), the influence of a periodic relief is negligible (i.e., the corrugated surface can be treated as a flat one), and, in order to find $n$ and $k$ by the least-squares method, a simpler formula to calculate the Fresnel coefficients can be used.

Optical modeling performed at successive stages of the processing can help to achieve the "tuning effect" to obtain the maximal sensitivity for the characteristic spectral region. These characteristics must be taken into account when designing constituents of the SPP-based devices to attain their maximum sensitivity.

\section{Results and discussions}

5.1. Optical properties of the layers deposited on a flat substrate

\subsubsection{GaAs native oxide}

In practice, even under considerably long etching after removal of the intrinsic oxide, we never obtain a bare GaAs. As soon as the sample after chemical treatment is exposed to air, a new oxide starts to form and grows during the SE measurements. Despite the care we took for the ultrahigh vacuum under evaporation conditions, the minor oxidation of the GaAs substrate processed cannot be avoided. This was taken into account in our calculations.

The ellipsometric analysis under the assumption of a three-phase (air/ intrinsic oxide / bulk GaAs) model by solving the Fresnel's equation was performed on the point-by-point (wavelength-by-wavelength) basis using ellipsometric data ( $\psi$ and $\Delta$ measured). The oxide overlayer thickness was numerically determined by minimizing the correlations between fit parameters with a linear regression analysis program [17]. The optical constants of GaAs and its intrinsic oxide used in the analysis are taken from [18] (GaAs) and from [19] (GaAs oxide), respectively. The oxide thickness is modeled as the equivalent dielectric layer of GaAs oxide, including a possible surface microroughness (i.e., it is not a real thickness, but an apparent oxide thickness). For simplicity, the thickness of the intrinsic oxide layer was fixed in the subsequent ellipsometric analysis of other structures considered in the present paper. The real surface of a sample can be described as the GaAs substrate covered with a rough oxide layer of $26 \AA$ in thickness.

\subsubsection{Dielectric films $-\mathrm{SiO}_{x}$}

Films of $\mathrm{SiO}_{\mathrm{x}}$ were prepared by vacuum evaporation of $99 \%$ pure silicon monoxide $(\mathrm{SiO})$ at a rate of $15 \mathrm{~nm} / \mathrm{s}$ (slow evaporation). A removable shutter was used to prevent the contamination of the substrate during the initial heating and outgassing of the vapour source. The pressure was $\sim 10^{-5}$ Torr before evaporation. Substrates were kept at RT during deposition [20]. Two kinds of substrates were used for evaporation and VASE measurements: a flat GaAs sample (etched for a short time) and a microscope glass slide.

These films, which were produced by slow evaporation of $\mathrm{SiO}$ in the presence of some amount of oxygen, do not have an exactly known stoichiometry. The oxygen content varies widely depending on the evaporation conditions $(0<x<2)$. Growth conditions of $\mathrm{SiO}_{\mathrm{x}}$ are expected to give thermodynamically nonequilibrium mixtures on the atomic scale. The structure, as suggested in [21], may be regarded as a random mixture of subunits that are Si-centered polyhedra of the types $\mathrm{Si}_{-} \mathrm{Si}_{4}, \mathrm{Si}_{-} \mathrm{Si}_{3} \mathrm{O}, \ldots, \mathrm{Si}_{-} \mathrm{O}_{4}$. The $\mathrm{SiO}_{\mathrm{x}}$ films produced under the above deposition conditions have been assumed to have the atomic ratio near 1.5.
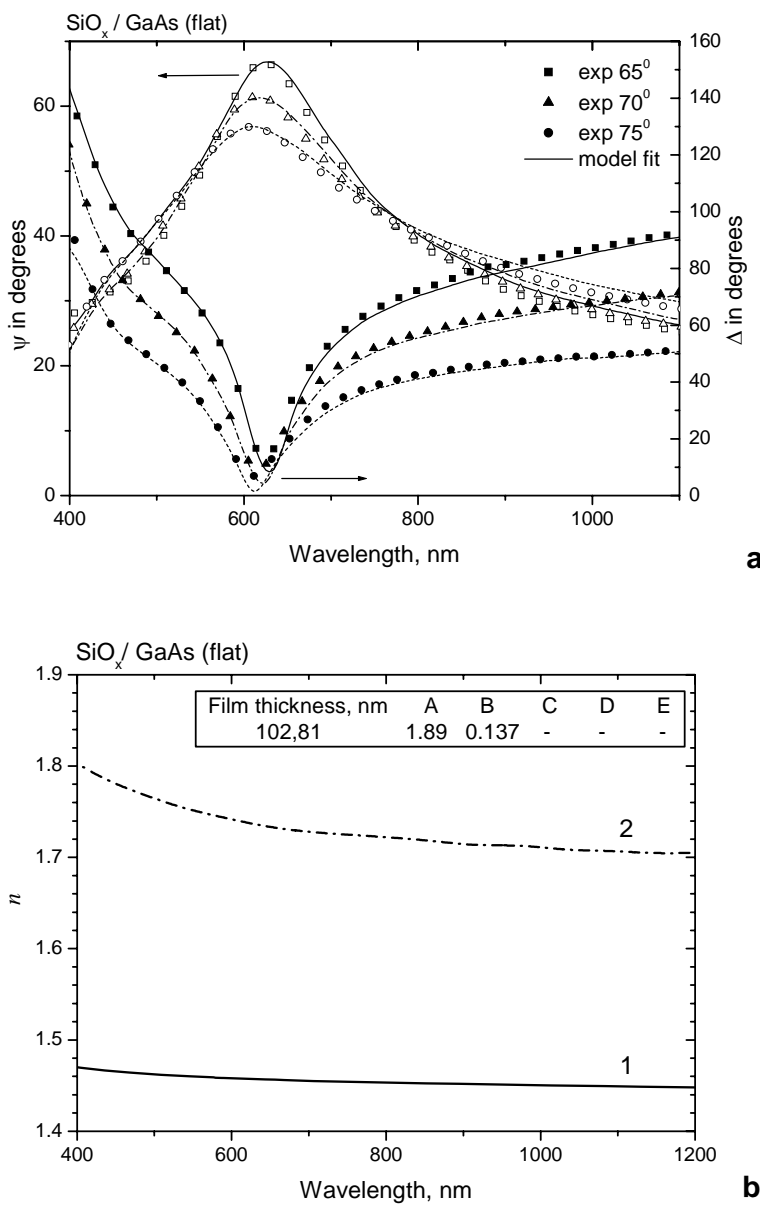

Fig. 5. $a$ - regression analysis of measured SE data for an asdeposited thermally evaporated $\mathrm{SiO}_{\mathrm{x}}$ film on the GaAs flat substrate. $b$ - refractive indices spectra for a $\mathrm{SiO}_{\mathrm{x}}$ film: (1) - best fit spectrum obtained on the basis of the Sellmeier model with parameters listed in the inset, (2) - spectrum is taken from [23]. 
The ellipsometric spectra of $\mathrm{SiO}_{\mathrm{x}} / \mathrm{GaAs}$ with the intrinsic oxide - flat structure are depicted in Fig. 5a. We considered the three-layer model of sample configuration in the simulation and the regression procedure because the interface between a deposited oxide film and underlying substrate is abrupt (as it was shown in [22]). The spectra cannot be interpreted with a simple assumption that the oxide is composed of a mixture of $\mathrm{Si}$ and $\mathrm{SiO}_{2}$ because of the different chemical nature of the film. Instead, the optical properties of the $\mathrm{SiO}_{x}$ film are determined by fitting its structure to the data, for which the optical constants were described by the Sellmeier relation (Eq. (2)).

In Fig. 5b, we present the spectrum of $n$ calculated with the Sellmeier parameters for the structure under study. It should be noted that the errors in the optical constants may arise as a result of errors in film thickness and substrate characterization. We believe that the measuring technique, in which the VASE technique combined with both the transmission of a thin film $\left(\mathrm{SiO}_{\mathrm{x}}\right)$ on the transparent substrate and the reflection of the same film on the GaAs substrate, is less influenced by such errors. Other errors may be introduced by deviations between the optical constants of the underlying layers with respect to the literature reference data. The coefficients $C, D$, and $E$ (Eq. (2)) appearing in the relation for the absorption coefficient are not shown because of their insignificantly small values (less than $10^{-4}$ ). There is no absorption in the UV-Vis - near-IR spectral regions. The refractive index of the evaporated $\mathrm{SiO}_{\mathrm{x}}$ film is consistently higher than the handbook value for a bulk $\mathrm{SiO}_{2}$ [23]. The formation of $\mathrm{Si}-\mathrm{Si}$ bonds has been demonstrated [21] to cause an increase of the refractive index and becomes more and more important (Si-rich oxide film formation) as the silicon content increases.

\subsubsection{Conductive oxide films - ITO}

To determine the optical constants and thickness of ITO films, a parametric representation of the spectra (Vis and near-IR) using the harmonic oscillator approximation was used by us earlier [24]. Taking into account that the ITO film is the material exhibiting some absorption, we divided the total film into two sublayers in order to use two sets of Lorentz oscillators (three oscillators) to model the ITO refractive index at the top and bottom of the film [25]. The best-fit optical constants at the top and bottom of the film prove that the extinction coefficient $k$ of the ITO film is lower at its bottom, i.e., the film is more conductive near the surface.

\subsubsection{Au island film on a glass}

In the present work, a combination of photometric and ellipsometric measurements in a common spectral range have been used. The advantage of the method is that the a priori knowledge of the optical constants is not need. The film thickness (equivalent to the coverage by dense
$\mathrm{Au}$ ) is determined independently (by a calibrated quartz crystal oscillator on the glass sample-witness). In Fig. 6a, we show the reflectance $R_{p}$ and transmittance $T_{p}$ data along with the regression fits based on the point-bypoint technique.

For the easier reference, the corresponding spectra of a glass sample-witness with $\mathrm{Au}$ island layer modeled using both the Bruggeman effective medium approximation and the point-by-point method were calculated (Fig. 6b). In the former method, the filling factor value was approximately taken from AFM results which show a definite island structure, assuming $50 \%$ $\mathrm{Au}$ and $50 \%$ voids. We found that the optical constants obtained by the both methods quite well agree with one another and differ from those of the bulk material [26].

\subsection{Optical properties of layers deposited on the corrugated substrate}

\subsubsection{Structure S1: ITO/GaAs (DG)}

We have analyzed the ellipsometric data (Fig. 7). The optical properties are conditioned by the microstructure of a film which is generally determined by deposition
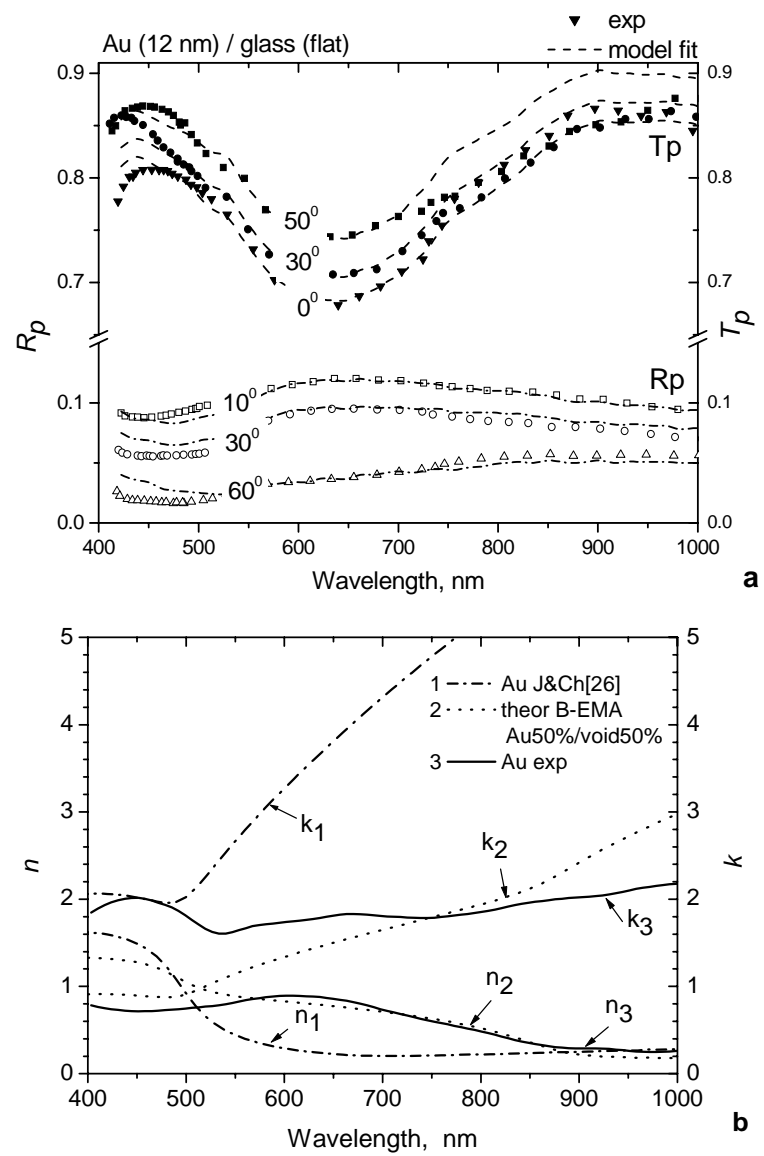

Fig. 6. $a-$ measured and calculated reflectance/ transmittance data for the 12-nm-thick Au island film deposited on the glass substrate. $b-A u$ film optical constants $n$ and $k$ obtained: in [26] (1), by best-fit using the B-EMA model (2) and from our VASE data by the point-by-point method (3). 


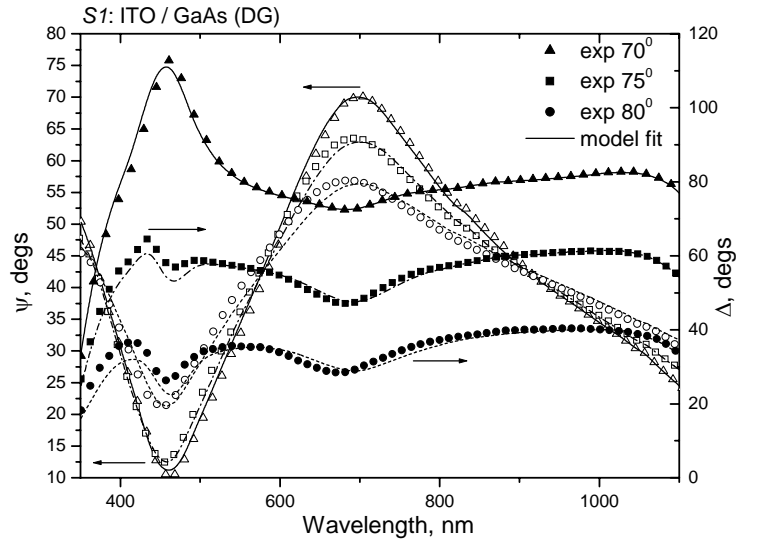

Fig. 7. Measured ellipsometric spectra (in case when grating vector is perpendicular to plane of incidence) and fitted spectra for sample $S 1$ (ITO/GaAs (DG)).

conditions. Ellipsometric analysis was performed by solving the Fresnel equations for $n$ and $k$ on the wavelength-by-wavelength basis. Inhomogeneity of the ITO film was inferred by us on the base of our SE measurements for the flat structure, although the specific nature and localization of the inhomogeneity was not deduced from the data. In the achievement of the fit, we used the results of our previous step of investigation (Sec. 5.1.3) obtained with the discrete description (graded structure). The results of analysis along with the previous results for comparison are shown in Fig 8. Values of $n$ and $k$ obtained by J.A. Woollam et al. on glass [27] are also shown as the reference values. Because the substrate was not bared (GaAs intrinsic oxide) and underwent the annealing procedure $\left(350^{\circ} \mathrm{C}\right.$, $30 \mathrm{~min}$ ), we supposed that the rather thick layer ITO $(160 \mathrm{~nm})$ is a continuous medium in its volume. It is also seen that the ITO film has noticeable absorption in the Vis. It was also reasonable to suppose that it has the inhomogeneous region (due to interdiffusion and chemical reactions) which is restricted mainly to the ITO/GaAs interface region. The ITO stoichiometry modification, as it was shown after [28], is mainly due to a variation of the parameters of the deposition process (the sputtering power and deposition rate). Since the above-mentioned technological factors are kept constant, the water content in the residual gas can be considered as a source of the deterioration of the ITO optical properties, and its role in the initial process of deposition and interface formation for the corrugated (diffraction grating) and flat surfaces of samples must be taken account. By comparing the changes in the optical constants $n$ and $k$ relative to those of ITO both deposited on a flat GaAs substrate and known from the literature (Fig. 8), we find that process-dependent changes in the optical constants in the Vis are quite evident. For a more detailed investigation of the ITO film microstructure, the additional analytic technique is required, but it is beyond the scope of this work.

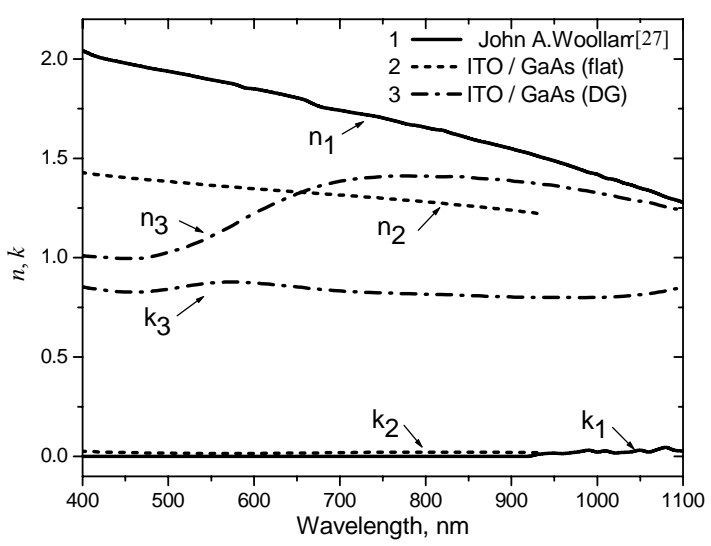

Fig. 8. ITO optical constants $n$ and $k$ obtained: by J.A. Woollam et al. (1), our previous results [24] with the use of the B-EMA model (2), from VASE data (3).

\subsubsection{Structures with $A u$ island top layers: $A u$ /GaAs (DG (S2)), $\mathrm{Au} / \mathrm{ITO} / \mathrm{GaAs}$ (DG) (S3).}

We believe that, all other things being equal (evaporation of the $\mathrm{Au}$ island film in manufacturing the structures S2 and S3 was performed under identical conditions), a different microstructure (porosity, roughness, etc.) of the underlying substrate used seems critical to provide the nucleation sites on the substrate surface and allows one to tailor a variety of final microstructures. In this context, the most essential difference between the samples is the narrower histogram of the distribution by the in-depth analysis of $S 3$ as compared with that of $S 2$ (Fig. 3). If the underlying surface $\left(R_{a}, r m s\right.$ - see Table 2$)$ is more smooth, then the more uniform distribution of $\mathrm{Au}$ islands on the surface occurs. The macroscopic optical properties must be affected by this fact.

From the SE spectra for the $\psi$ and $\Delta$ data (Fig. 9), the optical constants for $\mathrm{Au}$ films deposited on the different substrates are obtained by the above fitting (Fig. 10). For an $\mathrm{Au}$ (12-nm) film deposited on the GaAs DG (S2), the indices of island films are almost constant throughout a wide spectral range. Such a behavior is almost the same as that for an insulator - this state is approached by the island film when its lateral conductivity is interrupted by the partition of the metal layer into disconnected islands. It was found that, in the Vis region, the use of the ITO sublayer helps to reduce the absorption in the $\mathrm{Au}$ island film. In the near-IR region, however, a small increase in the absorption of the Au film deposited on DG covered by the ITO layer was observed (Fig. 10). To account for the difference in metal optical constants depending on the adjacent medium, several mechanisms can be considered. For example, if there are voids in the film, the measured dielectric constant will differ from the bulk one, depending on the volume of voids. 

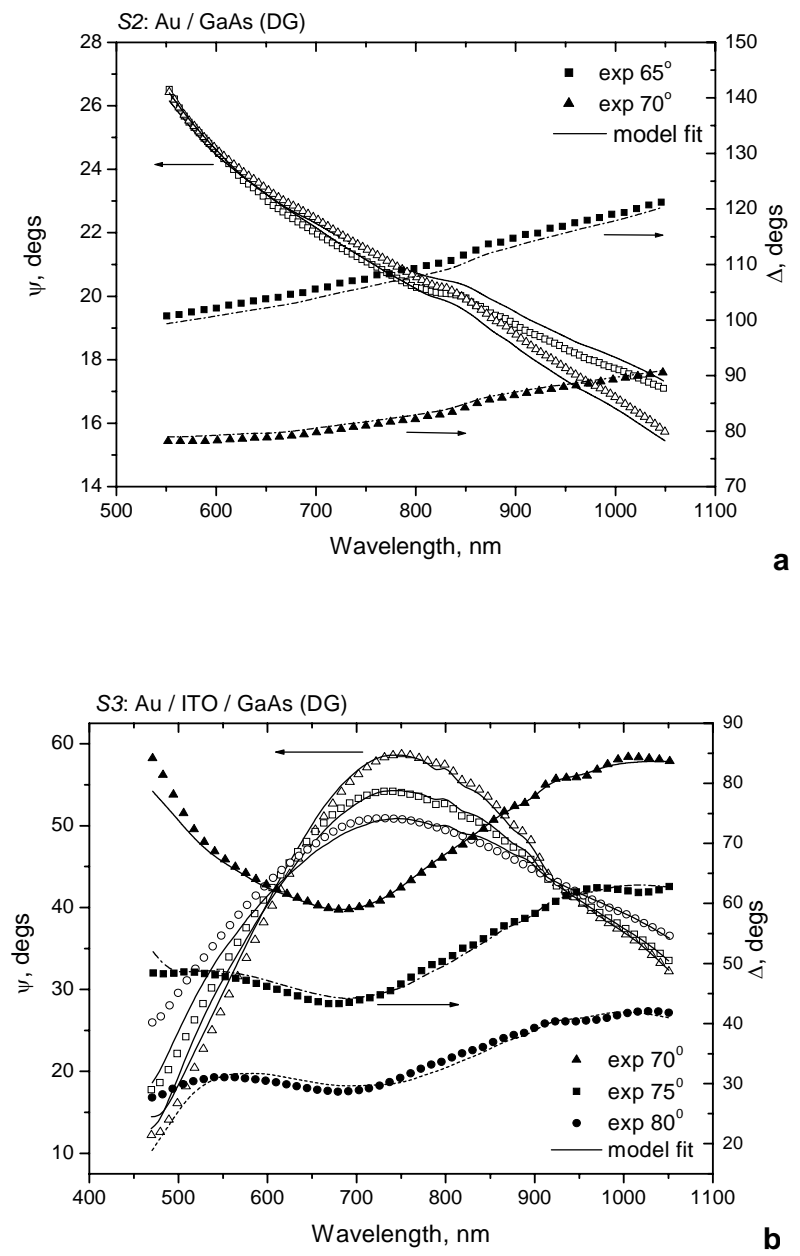

Fig. 9. Measured ellipsometric spectra (in the case where the grating vector is perpendicular to the plane of incidence) and the fitting for multilayer structures: $S 2(\mathrm{Au} / \mathrm{GaAs}(\mathrm{DG}))$ (a) and S3 (Au/ITO/GaAs (DG)) (b).

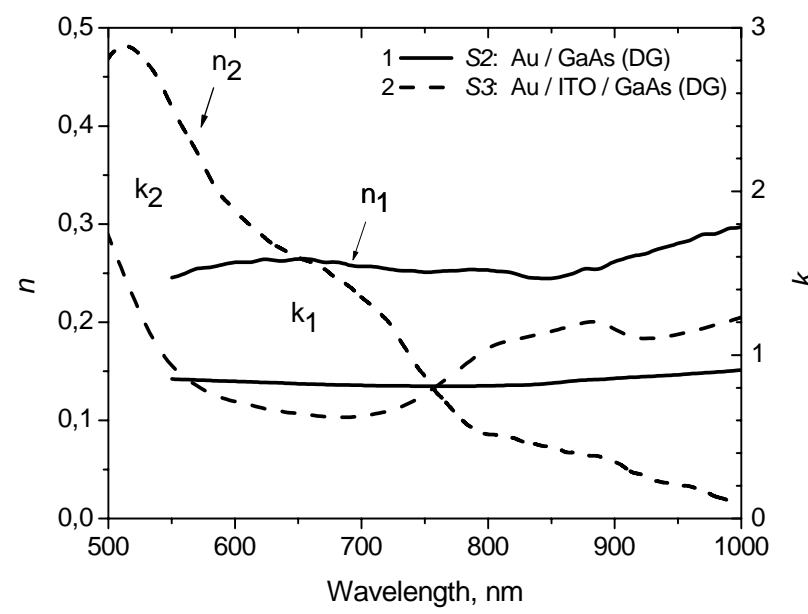

Fig. 10. Spectroscopic best-fit data obtained by the point-bypoint method for the refractive index $(n)$ and the absorption coefficient $(k)$ for $\mathrm{Au}(12-\mathrm{nm})$ films evaporated on different substrates.
The Au film thickness for all samples is near the percolation threshold. Therefore, even small changes in the surface morphology lead to significant changes in the optical properties. It is shown (Fig. 6b) that, for flat samples, the film with low filling factor is obtained. For an Au film deposited on GaAs DG (S2), the $n$ and $k$ indices of island films are almost constant throughout a wide spectral range. They are very similar to ones of the flat sample but with lower filling factor, because the additional relief of a diffraction grating produces the larger surface being covered by the film.

The ITO film of S1 and S3 makes the surface more smooth, by covering small pinholes and by hiding the relief peculiarities. It is shown in Fig. 3 that the ITO/GaAs (S1) sample has lowest relief height (and lowest surface area) comparing to those of $\mathrm{Au} / \mathrm{GaAs}$ (S2) and Au/ITO/GaAs (S3). Au was evaporated at $70^{\circ}$ to the surface normal, therefore only the surface apices were covered by the metal, and, as a result, the relief height was increased. Comparing S2 and S3, one can see that the relief height for $S 3$ is lower because of the initially smoother surface.

Optical constants spectra of $\mathrm{Au}$ films generally follow the surface area according to the EMA model except one feature appeared on the optical constants spectrum of S3 within the wavelength interval from 750 to $900 \mathrm{~nm}$ (Fig. 9b). We suspect that these unusual optic properties are due to the influence of a geometrical arrangement of resonant Au nanoparticles. Excited local surface plasmons increase the extinction, and we observe it via the increased $k$ value in regular analyses.

The effect of the substrate is very evident. Complex surface topology under holographically etching is obtained in one chemical reaction step by choosing the beam intensity distribution and the etching solution in a suitable way. The micro- and nanoprocesses represent an analogue of the 2D structure formation. Whereas the resonance phenomenon for a single particle strongly depends on details of the particle shape as well as on the dielectric properties of the particle material and the surrounding medium, the individual plasmon resonance for arranged particles is additionally influenced by the electromagnetic particle interaction via their dipolar fields which interfere to form a collective response. The authors of [29] probe the influence of grating effects (the grating constants $d$ ) on plasmon excitations in gold nanoparticles. They found that the extinction spectra show strongly different bandwidths for various grating constants with the maximum effect for $d=500 \mathrm{~nm}$ for metal nanoparticles gratings on the ITO base. In our case, there are a set of the grating constants (see the results of Fourier analysis, Fig. 4) on the nanoscale along with the fundamental one. The inhomogeneous band broadening is a function of fluctuations in the particle shape as well. Furthermore, by translating the probe beam and repeating the ellipsometric measurements on the portion of a sample with the 3-nm-thick $\mathrm{Au}$ island film (S4), we found the effects of not only the blue shift but also a more shallow resonance. This 
corroborates our finding that a shift and a broadening of the plasmon resonance are determined by the periodicity, size, and shape of $\mathrm{Au}$ aggregates. The detailed investigation of the effect will performed in near future.

\section{Conclusions}

This article describes the methodology and the results of the optical investigations of multilayer thin film structures on the surface relief (a diffraction grating) with regard for the outlook of their applications to plasmonics:

When taking into account the dependence of the optical parameters of the films on both deposition conditions and substrate peculiarities, the data obtained on the transparent or opaque but certainly flat witness samples must be taken to obtain the zero approximation for ones only;

- Both the SE and transmittance/reflectance data should be fitted, by using the parametrization of the dielectric function of a film material, rather than with the point-by-point method, because the former procedure reduces the number of unknown parameters.

- $\quad$ SE measurements on DG must be performed under conditions when the plane of incidence of light is parallel to the direction of grooves; in other case, one needs to consider the anisotropy of a Jones matrix [30].

- When solving the inverse ellipsometric (spectroscopic) problem for DG, one needs the fitting on these films, substrates, and overlayers, which were characterized on the planar ones obtained via one technological run.

- $\quad$ Results of measurements of the SPP parameters on the device structures of plasmonics give rise conveniently to the monitor quality of the optical characterization.

- Concerning the multilayer plasmonic structures with gold, ITO, and $\mathrm{SiO}_{\mathrm{x}}$ films, the result obtained on the surface plasmon polariton system as a candidate of optochemical sensor will be published in the next paper.

\section{References}

1. R.D. Harris, J.S. Wilkinson, Waveguide surface plasmon resonance sensors // Sensors and Actuators B 29, p. 261-267 (1995).

2. Martin N. Weiss, Ramakant Srivastava, Hovart Groger, Peter Lo, Shu-Fang Luo, A theoretical investigation of environmental monitoring using surface plasmon resonance waveguide sensors // Sensors and Actuators A 51, p. 211-217 (1996).

3. N.L. Dmitruk, O.I. Mayeva, S.V. Mamykin, O.B. Yastrubchak, and M. Klopflesch, Characterization and application of multilayer diffraction gratings as optochemical sensors // Sensors and Actuators A 88, p. $52-57$ (2001).
4. N.L. Dmitruk, O.I. Mayeva, S.V. Mamykin, I.B. Mamontova, O.B. Yastrubchak, Contribution of structure and morphology of design constituents to performance improvement of multilayer polaritonic photodetector // Sensors 3, p. 480-490 (2003).

5. N.L. Dmitruk, M. Klopfleisch, O.I. Mayeva, S.V. Mamykin, E.F. Venger and O.B. Yastrubchak, Multilayer diffraction gratings $\mathrm{Al} / \mathrm{GaAs}$ as polaritonic photodetectors // Phys. status solidi (a) 184, p. 165-174 (2001).

6. M. Quinten, U. Kreibig, Absorption and elastic scattering of light by particle aggregates // Appl. Opt. 6, p. 6173-6182 (1993).

7. Allan J. Waldorf, J.A. Dobrowolski, Brian T. Sullivan, L.M. Plante, Optical coating deposited by reactive ion plating // Appl. Opt. 32, p. 5583-5593 (1993).

8. K.H. Guenther, Physical and chemical aspects in the application of thin films on optical elements // Appl. Opt. 23, p. 3612-3632 (1984).

9. N.L. Dmitruk, O.Yu. Borkovskaya, O.I. Mayeva, I.B. Mamontova, Ultraviolet photodetector on the basis of heterojunction with textured interface // Sensors and Actuators A, 75, p. 151-155 (1999).

10. R.M.A. Azzam, N.M. Bashara, Ellipsometry and polarized light. Nebraska, Engineering Center, Lincoln, USA (1976).

11. E.F. Venger, A.V. Goncharenko, M.L. Dmitruk, Optics of Small Particles and Disperse Media. Naukova dumka, Kyiv, 1999 (in Ukrainian).

12. D.A.G. Bruggeman, Berechnung verschiedener physikalischer konstanten von heterogenen substanzen // Ann. Phys. (Leipzig) B 24, p. 636-674 (1935).

13. S. Spiga, G. Tallarida, A. Borghesi, A. Sassella, G. De Santi, Annealing effects on silicon-rich oxide films studied by spectroscopic ellipsometry // Thin Solid Films 325, p. 36-41 (1998).

14. C.F. Bohren, and D.R. Huffman, Absorption and Scattering of Light by Small Particles. WileyInterscience, New York, 1983.

15. Piotr J. Flatau, Improvements in the discrete-dipole approximation method of computing scattering and absorption // Optics Letters 22, No.16, p. 12051207 (1997).

16. J. Chandezon, M.T. Dupuis, G. Cornet, D. Maystre, Multicoated gratings: a differential formalism applicable in the entire optical region // J. Opt. Soc. Amer. 72, p. 839-846 (1982).

17. E.A. Irene, Applications of spectroscopic ellipsometry to microelectronics // Thin Solid Films 233, p. 96-111 (1993).

18. D.E. Aspnes and A.A. Studna, Dielectric functions and optical parameters of $\mathrm{Si}, \mathrm{Ge}, \mathrm{GaAs}, \mathrm{GaSb}, \mathrm{InP}$, InAs, and InSb from 1.5 to $6.0 \mathrm{eV} / /$ Phys. Rev. B 27, p. 985-1009 (1983).

19. Huade Yao, and Paul G. Snyder, In situ ellipsometric studies of optical and surface 
properties of GaAs (100) at elevated temperatures // Thin Solid Films 206, p. 283-287 (1991).

20. V.A. Dan'ko, I.Z. Indutnyy, I.Y. Maidanchuk, V.I. Min'ko, P.E. Shepelavyi and V.A. Yukhimchuk, Formation of the photoluminescence structure based on $\mathrm{SiO}_{\mathrm{x}}$ porous films // Optoelectronics and Semiconductor Techniques 39, p. 65-72 (2004) (in Russian).

21. H.R. Philipp, Optical properties of non-crystalline $\mathrm{Si}, \mathrm{SiO}, \mathrm{SiO}_{x}$ and $\mathrm{SiO}_{2} / /$ J. Phys. Chem. Solids 32 , p. 1935-1945 (1971).

22. Min Bai, and Fabian Pease, Electron beam induced conductivity in polymethylmethacrylate, polyamide, and $\mathrm{SiO}_{2}$ thin film // J. Vac. Sci. Technol. B 22 (6), p. 2907-2911 (2004).

23. E.D. Palik (Ed.), Handbook of Optical Constants of Solids. Academic Press, Orlando (Fl.), 1985.

24. M.V. Sosnova, N.L. Dmitruk, O.I. Mayeva, S.V. Mamykin, O.B. Yastrubchak, V.I. Min'ko, Multilayer waveguide structures based on diffraction gratings on GaAs for sensor application // Optoelectronic Information-Power Technologies. 1(9), p. 178-182 (2005).
25. R.A. Synovicki, Spectroscopic ellipsometry characterization of indium tin oxide film microstructure and optical constants // Thin Solid Films 313-314, p. 394-397 (1998).

26. P.B. Johnson, R.W. Christy, Optical constants of the noble metal // Phys. Rev. B 6, p. 4370-4379 (1972).

27. J.A. Woollam, W.A. McGahan, and B. Johs, Spectroscopic ellipsometry studies of indium tin oxide and other flat panel display multilayer materials // Thin Solid Films 241, p. 44-46 (1994).

28. S. Ishibashi, Y. Higuchi, Y. Ota, and K. Nakamura, Low resistivity indium tin oxide transparent conductive films. II. Effect of sputtering voltage on electrical property of films // J. Vac. Sci. Technol. A8(3), p. 1403-1406 (1990).

29. B. Lampreht, G. Schider, R.T. Lechner, H. Diltbacher, J.R. Krenn, A. Leitner, and F.R. Aussenegg, Metal nanoparticle gratings: Influence of dipolar particle interaction on the plasmon resonance // Phys. Rev. Lett. 84, p. 4721-4724 (2000).

30. R.M.A. Azzame and N.M. Bashara, Generalized ellipsometry for surfaces with directional preference: Application to diffraction gratings // J. Opt. Soc. Amer. 62, p. 1521-1523 (1972). 